\title{
A CPCH Access Method for Prioritized Services in W-CDMA
}

\author{
MoonYoung Choi, Member, IEEE, Yu-Dong Yao, Senior Member, IEEE, and Harry Heffes, Fellow, IEEE
}

\begin{abstract}
Common packet channel (CPCH) access is an efficient approach to support packet data transmissions in a wideband code-division multiple-access (W-CDMA) system. This letter introduces a simple access control method for $\mathrm{CPCH}$, which results in higher throughput. This method also provides prioritized services for different traffic classes. Each traffic class is assigned a distinct transmit permission probability that is determined at the new call initiation stage based on the status of CPCH channel occupancy. The differentiated service qualities, which correspond to different transmit permission probabilities, are evaluated in terms of packet blocking rates. The overall system performance is also evaluated in terms of normalized throughput.
\end{abstract}

Index Terms-Access control, channel assignment, common packet channel (CPCH), wideband code-division multiple-access (W-CDMA).

\section{INTRODUCTION}

A COMMON packet channel $(\mathrm{CPCH})$ is an uplink transport channel in the packet mode of wideband code division multiple access (W-CDMA) proposed by the 3rd generation partnership project (3GPP) [1]. It delivers small and medium size application messages (such as short message services, e-mails and web requests). The access protocol can be described as digital sense multiple access with collision resolution (DSMA-CR) [2]. Many CPCH access schemes have been investigated to improve the operation performance of $\mathrm{CPCH}$ [3]-[5]. There are three well-known access schemes for $\mathrm{CPCH}$, a basic scheme, a channel monitoring scheme (CM) and a channel assignment scheme (CA) [5]. In all three $\mathrm{CPCH}$ access procedures, an access phase (AP) and a collision detection (CD) phase are preceded before a packet transmission phase to reduce potential collisions of newly arrived packets. In [5], the performance of the three $\mathrm{CPCH}$ access schemes are evaluated and concluded that CA provides the best performance.

Recently, [6] proposed an access scheme with transmit permission probabilities so that multiaccess interference in the system is reduced. It finds an offered traffic load, $G_{\max }$, which maximizes the system throughput. It then maintains the

Manuscript received May 31, 2003. The associate editor coordinating the review of this letter and approving it for publication was Prof. P. Demestichas.

M. Choi is with Connectivity Laboratory, Digital Media R\&D Center, Samsung Electronics Company, Gyeonggi-Do 442-742, Korea (e-mail: moonyoung.choi@samsung.com).

Y.-D. Yao is with Wireless Information Systems Engineering Laboratory (WISELAB), Department of Electrical and Computer Engineering, Steven Institute of Technology, Hoboken, NJ 07030 USA (e-mail: yyao@ stevens.edu).

H. Heffes is with Department of Electrical and Computer Engineering, Stevens Institute of Technology, Hoboken, NJ 07030 USA (e-mail: hheffes@stevens.edu).

Digital Object Identifier 10.1109/LCOMM.2003.822498

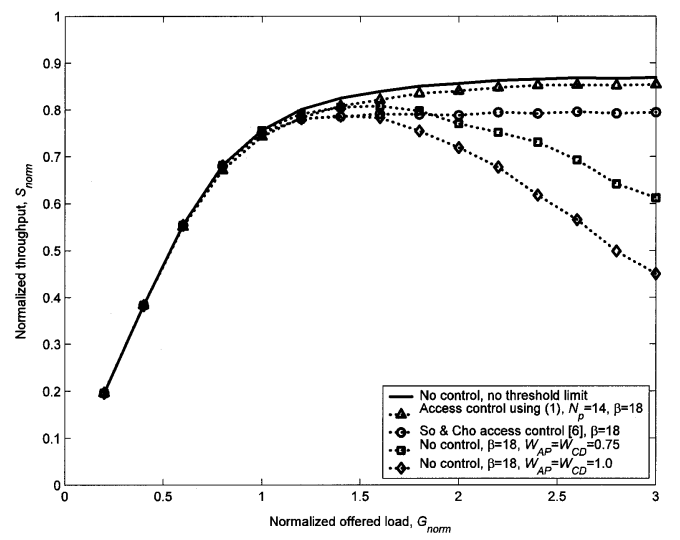

Fig. 1. Normalized throughput of $\mathrm{CPCH}$ access with or without access control. $\left(N_{c}=N_{\mathrm{AP}}=N_{\mathrm{CD}}=16, D=4\right.$, and $W_{\mathrm{AP}}=W_{\mathrm{CD}}=1.0$ unless otherwise specified in the figure).

maximum throughput through a permission probability which keeps the offered traffic load below $G_{\max }$ at any given time.

In this paper, we introduce a simple method to determine the permission probabilities and, furthermore, the permission probabilities are used to provide prioritized services for different traffic types in $\mathrm{CPCH}$. The differentiated service qualities are evaluated in terms of packet blocking rates and the system is also evaluated in terms of throughput.

\section{CPCH ACCESS, Multi-ACCESS INTERFERENCE, AND PERMISSION PROBABILITY}

Although there are several proposed $\mathrm{CPCH}$ access schemes, the channel assignment with channel status monitoring (CA-CM) is specified in the 3GPP document [7]. We will focus our study considering this access approach. When a call is initiated, an user equipment (UE) checks the availability of $\mathrm{CPCH}$ channels. If there is at least one free channel, the UE selects a signature corresponding to one of the free $\mathrm{CPCH}$ channels and transmits an AP preamble within an access time slot. In case all channels are occupied, the call will be backed off and a new call attempt will be initiated later. In AP, among the multiple call attempts from different users (using the same or different signatures) within an access time slot, only one signature will get a positive acknowledgment (ACK). An UE or UEs that receive the ACK will proceed to a CD phase. Each UE in CD selects one signature again and Node B (similar to a base station) acknowledges only one signature among all received $\mathrm{CD}$ signatures within one slot duration. $\mathrm{CD}$ signature sets may or may not be the same as AP signature sets. The UEs receiving ACKs in the CD phase transmit their packets through a channel 


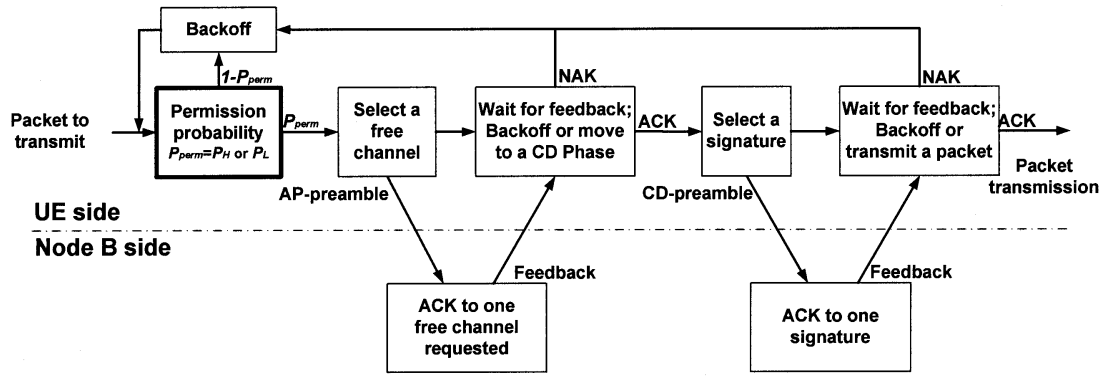

Fig. 2. The operational flow diagram of $\mathrm{CPCH}$ access for prioritized services.

originally determined in AP. In case two or more UEs get ACK, a collision will occur during their packet transmissions.

In wireless communications with code division multiple access (CDMA), multiaccess interference deteriorates the overall system performance. It is known that there is a certain level of multiaccess interference which a CDMA system can tolerate so that once the interference exceeds a threshold (say, $\beta$ ), the performance of packet transmissions degrades significantly [6]. The interference is caused by the simultaneous transmissions of packets in the system. The normalized throughput of $\mathrm{CPCH}$ access (CA-CM) to examine the impact of multiaccess interference is shown in Fig. 1 based on simulation results. In the simulation, we assumed that the number of $\mathrm{CPCH}$ channels in the system, $N_{c}$, is 16 , the number of AP signatures, $N_{\mathrm{AP}}$, is 16 , the number of CD signatures, $N_{\mathrm{CD}}$, is 16 , and the time duration from sending a preamble to receiving an acknowledgment for that preamble, $D$, is 4 access slots. We also assumed that the access slot time is of $1.33 \mathrm{~ms}$. and the default packet length is of $100 \mathrm{~ms}$. The solid line relates to an ideal scenario in which the throughput is limited by the number of $\mathrm{CPCH}$ channels and $\mathrm{AP} / \mathrm{CD}$ signatures in the system, not by the multiaccess interference. To consider the impact of multiaccess, we assume that all packet transmissions will fail when the total number of simultaneous transmissions exceeds $\beta$. The contribution to the multiaccess interference from an AP or CD preamble may be equal to or smaller than that from a message transmission packet. We use weight factors $W_{\mathrm{AP}}$ and $W_{\mathrm{CD}}$ to quantify this implication. In Fig. 1, the dotted line with square symbols assumes a case with $W_{\mathrm{AP}}=W_{\mathrm{CD}}=0.75$ and the dotted line with diamond symbols assumes $W_{\mathrm{AP}}=W_{\mathrm{CD}}=1$. It is seen from Fig. 1 that the throughput drops dramatically after a certain normalized offered load due to the multiaccess interference.

A permission probability can be used to reduce multiaccess interference. We examine the status of $\mathrm{CPCH}$ channel occupancy at each new call initiation. The permission probability is set to be 1 until the number of busy channels reaches a predefined number, $N_{p}$. Beyond this number the system starts access control using a permission probability smaller than 1 . The permission probability, $P_{\text {perm }}$, is formulated as follows,

$$
P_{\text {perm }}= \begin{cases}1 & N_{i}<N_{p} \\ \frac{N_{c}-N_{i}}{N_{c}-N_{p}} & N_{i} \geq N_{p}\end{cases}
$$

where $N_{i}$ is the number of busy channels. This describes a simple method determining permission probabilities.

In Fig. 1, throughput results of $\mathrm{CPCH}$ access with permission probabilities are plotted. The access control approach using (1) gives better performance comparing to a scheme proposed in [6] under high traffic load conditions (when $G_{\text {norm }}$ is approximately 1.2 in the figure). When the traffic load is lower, the scheme defined in (1) performs similarly to the scheme in [6]. Compared to the ideal scenario where the multiaccess interference is ignored, the throughput of our scheme approaches $97 \%$ or higher of the maximum throughput possible. This implies that the impact of the multiaccess interference is very small when the permission probability approach defined in (1) is applied. Note that the access control method specified in (1) can be easily implemented by monitoring the number of busy channels. The parameter $N_{p}$ is configurable based on system parameters such as $N_{c}$ and $\beta$.

\section{ACCESS SCHEME FOR PRIORITIZED SERVICES}

$\mathrm{CPCH}$ with CA-CM, together with the access control method described above, is to be considered for a system to support prioritized services. Two traffic classes are first considered here and different permission probabilities are applied to provide prioritized services. Fig. 2 shows the operation procedure. $P_{H}$ and $P_{L}$ in the figure represent the permission probability for highpriority traffic and that for low-priority traffic, respectively. We first choose the value $P_{H}$ as $\min \left\{\alpha P_{\text {perm }}, 1\right\}$, where $\alpha, \alpha \geq 1$, is a scaling factor. The next step is to determine $P_{L}$ using the following equation to maintain the same overall effective arrival rate (considering arrivals permitted entering the AP phase) compared to that for nonprioritized services:

$$
\lambda_{H} P_{H}+\lambda_{L} P_{L}=\left(\lambda_{H}+\lambda_{L}\right) P_{\text {perm }}
$$

where $\lambda_{H}$ and $\lambda_{L}$ are the arrival rates for high-priority traffic and low-priority traffic, respectively.

The normalized offered load, $G_{\text {norm }}$, and throughput, $S_{\text {norm }}$, are obtained as follows,

$$
\begin{aligned}
G_{\text {norm }} & =\frac{G_{H}+G_{L}}{N_{c}} \\
S_{\text {norm }} & =\frac{G_{H}\left(1-B_{H}\right)+G_{L}\left(1-B_{L}\right)}{N_{c}}
\end{aligned}
$$

where $G_{H}$ and $G_{L}$ are the offered loads of the high priority traffic and low-priority traffic. Similarly, $B_{H}$ and $B_{L}$ are the blocking probabilities for the two traffic types. The prioritized operation is simulated and the performance results are presented in Fig. 3. System parameters similar to those in Fig. 1 are used. We also consider that the high-priority traffic is of $70 \%$ of the total traffic $\left(\lambda_{L} / \lambda_{H}=3 / 7\right)$. Blocking probabilities for three 


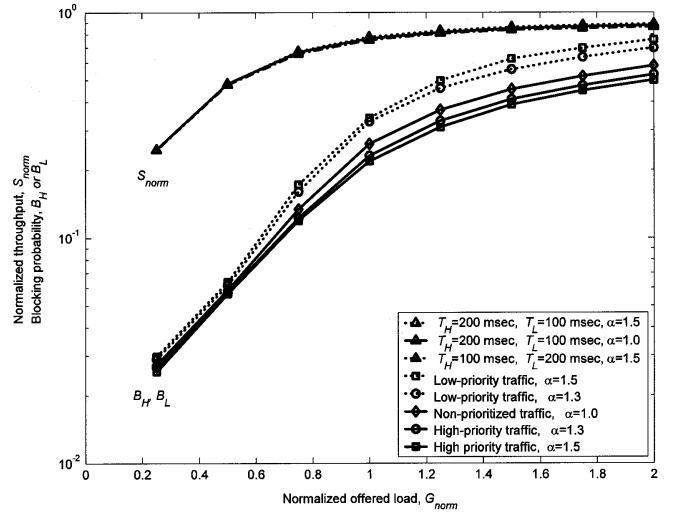

Fig. 3. Normalized throughput and blocking probabilities, impact of scaling factor $\alpha$ and packet length. $\left(N_{c}=N_{\mathrm{AP}}=N_{\mathrm{CD}}=16, D=4, W_{\mathrm{AP}}=\right.$ $W_{\mathrm{CD}}=1.0, \beta=18$, and $\left.\lambda_{L} / \lambda_{H}=3 / 7\right)$.

system scenarios are plotted in Fig. 3. The first scenario considers a single permission probability for both traffic classes so that they become nonprioritized $(\alpha=1)$. The second and the third cases assume priorities with $\alpha=1.3$ and $\alpha=1.5$, respectively. The results show that high-priority traffic achieves a lower blocking probability compared to low-priority traffic. There is a tradeoff between the two traffic types in blocking probabilities and this is adjusted through the scaling factor, $\alpha$. Normalized throughput results for the three cases are all found to be identical to that for the nonprioritized scenario as shown in Fig. 1 ("Access control using (1) with $N_{p}=14, \beta=18$ "). Different packet lengths are also considered for different traffic types, say, $T_{H}$ for high-priority traffic and $T_{L}$ for low-priority traffic, and simulation results show that the throughput differences are insignificant in all cases. Thus Fig. 3 indicates that, with access control, prioritized services are provided without sacrificing system throughput.

Note that, when there are $n$ traffic classes, permission probabilities for all traffic classes except the lowest-priority traffic class are selected based on their priority levels. The permission probability for the lowest-priority traffic class, $P_{L}$, is obtained by using the following equation:

$$
\sum_{i=1}^{n-1} \lambda_{i} P_{i}+\lambda_{L} P_{L}=\left(\sum_{i=1}^{n-1} \lambda_{i}+\lambda_{L}\right) P_{p e r m}
$$

where $\lambda_{i}$ and $P_{i}$ are the arrival rate and permission probability of the $i$ th traffic class.

\section{CONCLUSIONS}

A simple method is introduced to calculate permission probabilities based on the $\mathrm{CPCH}$ channel occupancy status, which is used to reduce multiaccess interference and keep normalized throughput very close to the ideal scenario where the multiaccess interference is not considered. Different permission probabilities are further used for different classes of traffic to achieve improved blocking performance for the high-priority traffic at the expense of degraded blocking performance for the low-priority traffic class.

\section{REFERENCES}

[1] K. Parsa, S. Ghassemzadeh, and S. Kazeminejad, "Systems engineering of data services in UMTS W-CDMA," in Proc. IEEE Int. Conf. of Communications (ICC2001), vol. 5, 2001, pp. 1373-1380.

[2] K. Parsa, "An overview of common packet channel $(\mathrm{CPCH})$, an optimum wireless internet mechanism in 3GPP W-CDMA system and comparison of various UMTS non real time data deployment options," in Proc. IEEE PIMRC, vol. 1, 2000, pp. 388-395.

[3] 3GPP TSG-RAN Working Group 1. (1999, Sept.) Performance of CPCH, TSGR1\#7(99)b36. Philips. [Online] Available: ftp://ftp. 3gpp.org

[4] 3GPP TSG-RAN Working Group 2. (1999, Nov.) Performance Evaluation of CPCH, TSGR2\#8(99)e13. Samsung. [Online] Available: ftp://ftp. 3gpp.org

[5] J. W. So and D. H. Cho, "Access scheme for integrated voice/data transmissions over common packet channel in 3GPP," IEEE Commun. Lett., vol. 5, pp. 46-48, Feb. 2001.

[6] _- "A new access scheme for common packet channel in W-CDMA system," in IEEE VTC - Spring, vol. 2, 2001, pp. 1042-1046.

[7] 3GPP TSG-RAN Working Group 2. (2001, Sept.) Medium access control (MAC) Protocol specification, TS25.321. [Online] Available: ftp://ftp. 3gpp.org 